\section{Risk factors for iatrogenic retinal breaks induced by separation of posterior hyaloid face during 23-gauge pars plana vitrectomy}

R Rahman ${ }^{1}$, CD Murray ${ }^{1}$ and J Stephenson²
${ }^{1}$ Department of Ophthalmology, Calderdale Royal Hospital, Halifax, West Yorkshire, UK

${ }^{2}$ School of Human and Health Sciences, University of Huddersfield,

Queensgate, Huddersfield, West Yorkshire, UK

Correspondence:

R Rahman, Department of Ophthalmology, Calderdale Royal Hospital, Salterhebble Hill, Halifax, West Yorkshire HX3 OPW, UK.

Tel: +44 (0)1422 222977

Fax: +44 (0)1422 222544

E-mail: Rubina.Rahman@

nhs.net

Received: 22 June 2012 Accepted in revised form: 15 January 2013 Published online: 1 March 2013

\begin{abstract}
Purpse To describe the incidence and features of intraoperative retinal breaks caused by induction of posterior hyaloid face (PHF) separation during 23-gauge pars plana vitrectomy (PPV).

Methods A prospective, consecutive, single surgeon, observational study of patients undergoing 23-gauge transconjunctival sutureless vitrectomy for macular pathology or floaters, was carried out between 2009 and 2011 at Calderdale Royal Hospital, UK. PHF separations were categorised as either suction induced or requiring membrane blue (DORC Limited) with suction (adherent). The outcome measure was dichotomised into retinal break or tear and no retinal break or tear. Forced entry and parsimonious multiple logistic regression analyses were conducted, using statistical software, to test for significance of association of the set of recorded factors and covariates with the dichotomised outcome measure.

Results Data were collected and analysed from 137 patients. The incidence of iatrogenic retinal breaks associated with PHF separation during 23-gauge PPV was $18.2 \%$. Under both forced entry and parsimonious multiple logistic regression models, the odds of a retinal break or tear reduce by about $3-4 \%$ for each increasing year of age. Findings also showed some substantive association in the forced entry model, with the odds of a retinal break or tear for adherent cases being around 3.8 times those for suctiononly cases.
\end{abstract}

Conclusions Mechanical detachment of the PHF represents an important risk factor in the formation of retinal breaks. Particular care should be taken to examine intraoperatively for iatrogenic breaks in order to prevent missed breaks and subsequent rhegmatogenous retinal detachments.

Eye (2013) 27, 652-656; doi:10.1038/eye.2013.6; published online 1 March 2013

Keywords: 23-gauge; sutureless; vitrectomy; iatrogenic; retinal; breaks

\section{Introduction}

The incidence and features of iatrogenic retinal breaks caused during pars plana vitrectomy (PPV) surgery is of paramount importance to both surgeon and patient. The natural progression from missed retinal break to postoperative retinal detachment is not only a strain on time and resources, but it may be deleterious to the final visual acuity of the individual patient. Intraoperative recognition and treatment of these breaks reduces the postoperative risk of retinal detachment to that among eyes without observed intraoperative breaks. ${ }^{1}$ The surgeon must perform with full understanding and knowledge of the limitations of instrumentation and machinery used in his or her hands. Equipped with this approach, iatrogenic retinal breaks can both be anticipated and promptly treated to the satisfaction of surgeon and patient.

The incidence of recognised peripheral retinal breaks discovered at 20-gauge PPV has been 
reported to be 4 to $11 \% \cdot{ }^{1-4}$ Moore et al found no statistically significant association between posterior vitreous detachment (PVD) induction and retinal breaks in a retrospective observational series. ${ }^{1}$ Tan et $a l^{5}$ reported a $15.8 \%$ incidence of intraoperative breaks in a series of 177 consecutive cases undergoing 25-gauge transconjunctival sutureless vitrectomy (TSV). In this series, a statistically significant relationship between PVD induction and the presence of breaks elsewhere was identified. ${ }^{5}$

The 23-gauge instruments allow the surgeon to combine the benefits of TSV (improved patient comfort and faster postoperative recovery) without a significant compromise on instrument performance and rigidity. ${ }^{6}$

The 23-gauge PPV is now the most popular type of TSV used for a whole range of vitreo-retinal pathology. ${ }^{7}$ The purpose of this study was to examine and report the incidence and features of intraoperative retinal breaks caused by induction of posterior hyaloid face (PHF) separation during 23-gauge PPV surgery.

\section{Materials and methods}

The study proposal was discussed with the local Research and Development department who decided that ethical approval was not required. The basis for this decision was made on the fact that there would be no change to the standard of care for this group of study patients. This was a prospective, single surgeon, consecutive, observational study of patients undergoing 23-gauge TSV carried out between 2009 and 2011 at Calderdale Royal Hospital, UK.

Baseline demographic data, indication for surgery, and axial length $(\mathrm{mm})$ were recorded for each patient. Spherical equivalent was recorded, but not used in subsequent analysis because of its high degree of collinearity with axial length. Inclusion criteria were patients with an attached PHF undergoing 23-gauge PPV for macula hole $(\mathrm{MH})$, vitreomacular traction (VMT), epiretinal membrane (ERM), or floaters. Preoperatively, dilated fundoscopy was performed to check for any preexisting peripheral retinal pathology. Attached PHF was confirmed intraoperatively at the time of PPV with the use of the BIOM (Oculus, Wetzlar, Germany) viewing system. In all cases, vitrectomy was performed by a three port pars plana approach with sclerotomies created $3.5 \mathrm{~mm}$ away from the limbus. A total of 119 patients underwent combined phakoemulsification and vitrectomy surgery while 18 patients were already pseudophakic at the time of vitrectomy. Separation of the PHF was induced by suction from the 23-gauge vitreous cutter probe and advanced up to the vitreous base. PHF separation was categorised as either suction induced, where light suction near the optic disc was sufficient to cause separation of the PHF, or adherent (requiring membrane blue with suction). In the latter, if PHF separation could not be achieved initially with light suction at the disc, membrane blue was then used to better identify the peripapillary vitreous to target suction and prevent inadvertent damage to peripapillary retina from continued suction in these cases.

The outcome measure was dichotomised into retinal break or tear and no retinal break or tear. Patients who had experienced a retinal haemorrhage were grouped with those patients categorised as not experiencing a retinal break or tear. Pre-existing retinal breaks were excluded for study purposes. A break was categorised as pre-existing if it was round and had substantial underlying pigment surrounding it. All intraoperative findings were recorded immediately after the procedure by the operating surgeon. These included any peripheral retinal pathology resulting directly from $\mathrm{PHF}$ detachment such as retinal break/tear and retinal haemorrhages. The surgeon also recorded any difficulty encountered in induction of PHF separation and the use of membrane blue to define the vitreo-papillary adhesion in these cases. Records included where persistent peripheral vitreous attachment was encountered, despite detachment at the disc. Treatment used to treat peripheral retinal breaks and postoperative incidence of rhegmatogenous retinal detachment (RRD) was also recorded. All patients in this study were followed for a minimum period of 6 months from surgery.

Forced entry and parsimonious multiple logistic regression analyses were conducted, using Statistical package for the social sciences (SPSS) version 18.0 statistical software (IBM, Portsmouth, UK), to test for significance of association of the set of recorded factors and covariates with the dichotomised outcome measure. The forced entry model assessed the significance of each parameter in a multiple generalised linear model in the presence of other parameters; whereas the parsimonious model was developed from the forced entry model to describe the data using a sub-set of the variables considered in this model as economically as possible. Parameter significance was assessed using a 5\% significance level. Odds ratios and associated 95\% confidence intervals for each assessed parameter were also derived, with primary inferences of significance being inferred from the parsimonious model.

The association between diagnosis and findings was assessed using the $\chi^{2}$ test for association, and the magnitude of the effect assessed using Cramer's V coefficient. The occurrence of break formation in each category of the diagnosis variable was also determined.

The location of all breaks was classified as either superior (between ' 8 o' clock' and ' 4 o' clock' measured in a clockwise direction) or inferior (between ' 4 o'clock' and '8 o'clock' measured in a clockwise direction). Patients 
for whom one or more retinal breaks or tears were recorded were classified as having either (a) break(s) in the superior region only; (b) breaks in the inferior region only; or (c) breaks in both superior and inferior regions. McNemar's test for association of dependent groups was conducted on the data to assess the hypothesis that the expectation of frequencies in the two regions was equal.

We certify that all applicable institutional and governmental regulations concerning the ethical use of human volunteers were followed during this research.

\section{Results}

Data were collected on 137 patients with ages ranging from 32 to 94 years. Mean age in years was 69.6 (SD 11.9). The mean axial length in mm was 23.7 (SD 1.41).

Preoperative diagnosis frequency was as follows: MH 71 (51.8\%), VMT 12 (8.8\%), ERM 27 (19.7\%), floaters 27

(19.7\%). Posterior hyaloid separation technique-suction only: $126(92.0 \%)$, suction and membrane blue (adherent): $10(7.3 \%)$, and missing: $1(0.7 \%)$. A total of 112 patients $(81.8 \%)$ were classified as having no retinal break or tear. In all, 25 (18.2\%) were classified as having a retinal break or tear. Of these 25 patients for whom a retinal break or tear was recorded, $13(52.0 \%)$ had a diagnosis of $\mathrm{MH}$, $2(8.0 \%)$ had a diagnosis of VMT, $3(12.0 \%)$ had a diagnosis of ERM, and $7(28.0 \%)$ had a diagnosis of floaters.

None of the assessed covariates achieved statistical significance under the forced entry model, with the greatest degree of substantive significance indicated by the age covariate. A parsimonious logistic regression model derived using a modelling strategy based on likelihood ratio considerations found age to be the only statistically significant covariate $(P=0.028)$. Hence under either model, at best estimate, the odds of a retinal break or tear reduce by about 3-4\% for each increasing year of age. Findings also showed some substantive association in the forced entry model, with the odds of a retinal break or tear for adherent cases being around 3.8 times those for suction-only cases at best estimate.

$P$-values, odds ratios, and confidence intervals for the multiple regression model are summarised in Table 1. $P$-values, odds ratios, and confidence intervals for the parsimonious regression model are summarised in Table 2.

McNemar's testing undertaken on the location of the breaks found evidence to suggest that retinal break/tears are not equally distributed across the eye $\left(\chi^{2}{ }_{(1)}=11.52\right.$, $P<0.001$ ), with 16 cases of eyes with a break/tear in the superior region only, and a single case of an eye with a break/tear in the inferior region only. In addition, 8 cases were reported with breaks in both regions, and 112 with breaks in neither region.
Table $1 P$-values, odds ratios, and 95\% confidence intervals: forced entry multiple logistic regression model

\begin{tabular}{lccc}
\hline Parameter & P-value & Odds ratio & $95 \%$ CI for OR \\
\hline Age & 0.131 & 0.967 & $(0.925,1.010)$ \\
Axial length & 0.917 & 1.018 & $(0.721,1.438)$ \\
Diagnosis & & & \\
$\quad$ MH (reference) & & & \\
$\quad$ VMT & 0.923 & 0.910 & $(0.134,6.173)$ \\
$\quad$ ERM & 0.244 & 0.411 & $(0.092,1.833)$ \\
$\quad$ Floaters & 0.817 & 1.159 & $(0.332,4.045)$ \\
Findings & & & \\
$\quad$ Suction only (reference) & & & \\
$\quad$ Adherent & 0.124 & 3.763 & $(0.696,20.34)$ \\
\hline
\end{tabular}

Table $2 P$-values, odds ratios, and 95\% confidence intervals: parsimonious logistic regression model

\begin{tabular}{lccc}
\hline Parameter & P-value & Odds ratio & 95\% CI for OR \\
\hline Age & 0.028 & 0.959 & $(0.924,0.996)$ \\
\hline
\end{tabular}

A statistically significant association between diagnosis and findings was found using the $\chi^{2}$ test for association $\left(\chi^{2}(3)=13.1, P=0.003\right)$. Cramer's V statistic was calculated to be $31.9 \%$, indicating an effect small-tomedium in magnitude. Although most observed frequencies were close to those expected under the null hypothesis of no association, all patients with a diagnosis of macular hole were associated with findings of suction only, leading to observed frequencies of patients with a macular hole diagnosis showing some discrepancy from corresponding expected frequencies.

\section{Discussion}

TSV surgery carries several risks including cataract, haemorrhage, raised intraocular pressure, hypotony, endopthalmitis, iatrogenic retinal breaks, and retinal detachment. Missed iatrogenic breaks during PPV inevitably result in RRD and further surgery. Consequently, the above-mentioned risks increase as well as the drive for proliferative vitreoretinopathy. The end result may be a deleterious effect on the patient's anatomical and visual outcome. ${ }^{8}$ The incidence of postvitrectomy retinal detachment was reported to be similar in small gauge vitrectomy $(1.7 \%)$ compared with 20 gauge surgery (1.2\%). ${ }^{9}$ This incidence may be dependent on several components including ocular comorbidity, surgical technique, and instrumentation used. A Moorfields Eye Hospital retrospective review of 645 20-gauge PPV cases reported 4 in 10 iatrogenic peripheral retinal breaks, which may be associated with traction at sclerotomy sites, occurring intraoperatively from 
instruments engaging the vitreous base during entry and exit movements. ${ }^{10}$ A large retrospective study of 2598 small-incision TSV (23 and 25-gauge) concluded that in most cases the RD was not caused by the sutureless technique itself, but was most likely related to the underlying pathology or new retinal tears not in the proximity of the sclerotomies. ${ }^{11}$ Le Rouic et al ${ }^{12}$ report a single-centre retrospective comparative study of 23-gauge (349 eyes) vs 20-gauge (346 eyes) vitrectomy macula surgery with regards to postoperative risk of RD. Iatrogenic intraoperative tears were detected and treated in $1.2 \%$ of the 23-gauge group and $1.4 \%$ of the 20-gauge group $(P=0.72)$. None of these cases developed postoperative RD. In our study, where all eyes initially had an attached $\mathrm{PHF}$, iatrogenic intraoperative tears were detected and treated in $18.2 \%$ (25/137). Although our study primarily described the incidence and features of intraoperative retinal breaks caused by induction of PHF separation during 23-gauge PPV surgery, none of the iatrogenic breaks were associated with 23-gauge sclerotomy sites.

Mechanical detachment of the PHF represents an important risk factor in the formation of retinal breaks, which significantly increases the risk of RRD in the postoperative period if left undetected. ${ }^{5}$ The technique of induction of PHF separation in our study was light suction near the optic disc on the whole and in $7.3 \%$ the posterior hyaloid was found to be more adherent, requiring membrane blue and precise suction on the peripapillary vitreous to prevent inadvertent damage to peripapillary retina from continued suction in these cases. Eyes noted intraoperatively to have a particularly adherent PHF were at 3.8 increased odds of iatrogenic breaks compared with those less adherent. In all cases and especially the latter, it was imperative to carry out a full 360-degree examination with indentation, to exclude any iatrogenic retinal breaks. In our study, there were no cases of immediate or late post-vitrectomy RRD, a finding consistent with Le Rouic et al. ${ }^{12}$ We suggest part of the reason for this is that the surgeon used combined phacoemulsification and PPV, which enabled an unimpeded view to the vitreous base. This allowed better shaving of the vitreous gel for a more extensive peripheral vitrectomy as well as better visualisation and prompt treatment of iatrogenic breaks.

Although one limitation may be the smaller numbers involved when compared with other larger retrospective studies, the strength of our study lies in the single surgeon prospective design, combined with examination of a large number of variables. This has resulted in robust and reliable results. None of the recorded covariates achieved statistical significance, however, patient age appeared to have some substantive significance associated with the risk of iatrogenic retinal breaks, with the odds of a retinal break or tear reducing by about
$3-4 \%$ for each increasing year of age. Although the diagnoses of VMT, ERM, and floaters showed close to no association with the observed frequencies, $\mathrm{MH}$ appeared to have some association with the PHF requiring 'suction only' to induce separation.

\section{Conclusion}

Mechanical detachment of the PHF represents an important risk factor in the formation of retinal breaks, which significantly increases the risk of RRD in the postoperative period if left undetected. This case series reports an $18.2 \%$ incidence of iatrogenic retinal breaks associated with PHF separation during 23-gauge PPV. The odds of a retinal break or tear were found to reduce by about $3-4 \%$ for each increasing year of age. Eyes noted intraoperatively to have particularly adherent PHFs are at around 3.8 increased odds of iatrogenic breaks compared with those less adherent. Particular care should be taken in these cases to examine intraoperatively for iatrogenic breaks in order to prevent missed breaks and subsequent RRDs.

\section{Summary}

What was known before

- Iatrogenic retinal breaks during 23-gauge TSV are thought to be related to a number of possible factors including: entry sites, underlying pathology, and induction of PHF separation.

What this study adds

- Separation of the PHF is a major risk factor for iatrogenic breaks during 23-gauge TSV for macula pathology and floaters. Two important related risk factors include: agewhere the odds of a retinal break or tear reduce by about $3-4 \%$ for each increasing year of age. PHF adherence-the odds of a retinal break or tear for adherent cases being around 3.8 times those for suction-only cases. We introduce a surgical classification of PHF adherence, which aids in identifying more high-risk cases.

\section{Conflict of interest}

The authors declare no conflict of interest.

\section{References}

1 Moore JK, Kitchens JW, Smiddy WE, Mavrofrides EC, Gregorio G. Retinal breaks observed during pars plana vitrectomy. Am J Ophthalmol 2007; 144: 32-36.

2 Carter JB, Michels RG, Glaser BM, De Bustros S. Iatrogenic breaks complicating pars plan vitrectomy. Ophthalmology 1990; 97: 848-853.

3 Sjaarda RN, Glaser BM, Thompson JT, Murphy RP, Hanham A. Distribution of iatrogenic retinal breaks in macular hole surgery. Ophthalmology 1995; 102: 1387-1392. 
4 Territo C, Geiser JP, Wilson CA, Anand R. Influence of the cannulated system on the occurrence of iatrogenic slerotomy retinal tears. Retina 1997; 17: 430-433.

5 Stevie Tan H, Mura M, De Smet MD. Iatrogenic retinal breaks in 25-gauge macular surgery. Am J Ophthalmol 2009; 148: $427-430$.

6 Hubschman JP, Gupta A, Bourla DH, Culiat M, Yu F, Schwartz SD. 20-, 23-, and 25-gauge vitreous cutters performance and charachteristics evaluation. Retina 2008; 28: 249-257.

7 Sisk RA, Murray TG. Combined phacoemulsification and sutureless 23-gauge pars plana vitrectomy for complex vitreo-retinal diseases. Br J Ophthalmol 2010; 94: 1028-1032.

8 Tabandeh H, Chaudhry NA, Smiddy WE. Retinal detachment associated with macula hole surgery. Retina 1999; 19: 281-286.
9 Rizzo S, Belting C, Genovesi-Ebert F, diBartolo E. Incidence of retinal detachment after small-incision, sutureless pars plana vitrectomy compared with conventional 20 gauge vitrectomy in macular hole and epiretinal membrane surgery. Retina 2010; 30: 1065-1071.

10 Ramkissoon YD, Aslam SA, Shah SP, Wong SC, Sullivan PM. Risk of iatrogenic peripheral retinal breaks in 20-G pars plana vitrectomy. Ophthalmol 2010; 117: 1825-1830.

11 Rizzo S, Belting C, Genovesi-Ebert F. Retinal detachment after small-incision, sutureless pars plana vitrectomy: possible causative agents. Graefes Arch Clin Exp Ophtalmol 2010; 248: 1401-1406.

12 Le Rouic J-F, Becquet F, Ducournau D. Does 23-gauge sutureless vitrectomy modify the risk of postoperative retinal detachment after macula surgery? A comparison with 20-gauge vitrectomy. Retina 2011; 31(5): 902-908. 